\title{
Right-To-Left Ventricular Differences in the Expression of Mitochondrial Hexokinase and Phosphorylation of Akt
}

\author{
Petra Waskova-Arnostova ${ }^{\mathrm{a}}$ Barbara Elsnicova ${ }^{\mathrm{a}}$ Dita Kasparova ${ }^{\mathrm{a}}$ Ondrej Sebesta ${ }^{\mathrm{b}}$ \\ Jiri Novotnya Jan Neckarc Frantisek Kolarc Jitka Zurmanova ${ }^{a}$
}

\begin{abstract}
aDepartment of Physiology and 'Laboratory of Confocal and Fluorescence Microscopy, Faculty of Science, Charles University in Prague, Prague; Institute of Physiology, Academy of Sciences of the Czech Republic, Prague
\end{abstract}

\section{Key Words}

Hexokinase isoforms $•$ Akt kinase $\bullet$ Left ventricle $\bullet$ Right ventricle $\bullet$ Mitochondria co-localization

\begin{abstract}
Background/Aims: Hexokinase (HK) is a key glycolytic enzyme which promotes the maintenance of glucose homeostasis in cardiomyocytes. HK1 isoform is predominantly bound to the outer mitochondrial membrane and highly supports oxidative phosphorylation by increasing the availability of ADP for complex $V$ of the respiratory chain. HK2 isoform is under physiological conditions predominantly localized in the cytosol and upon stimulation of PI3K/ Akt pathway associates with mitochondria and thus can prevent apoptosis. The purpose of this study was to investigate expression and subcellular localization of both HK isoforms in left (LV) and right (RV) heart ventricles of adult male Wistar rats. Methods: Real-Time RT-PCR, Western blotting, and quantitative immunofluorescence microscopy were used. Results: Our results showed a significantly higher expression of both HK1 and HK2 at mRNA and protein levels in the RV compared to the LV. These findings were corroborated by immunofluorescence staining which revealed substantially higher fluorescence signals of both HKs in the RV than in the LV. The ratios of phospho-Ser473-Akt/non-phospho-Akt and phospho-Thr308-Akt/nonphospho-Akt were also markedly higher in the RV than in the LV. Conclusion: These results suggest that the RV has a higher activity of aerobic glycolytic metabolism and may be able to respond faster and more powerfully to stressful stimuli than the LV.
\end{abstract}

Copyright (C) 2013 S. Karger AG, Basel 


\section{Introduction}

Hexokinase (HK) is a key glycolytic enzyme of the cardiac energy metabolism which keeps the intracellular glucose concentration at a low level by phosphorylating glucose to glucose-6-phosphate (G-6-P), thereby regulating glucose fluxes into cardiomyocytes. HK is essential for the regulation of glucose utilization and for the maintenance of glucose uptake [1]. Two HK isoforms (HK1 and HK2) present in cardiac tissue [2, 3] differ in their substrate affinity and subcellular localization reflecting their different metabolic roles [4-6]. Under physiological conditions, HK1 is predominantly bound to the outer mitochondrial membrane, where it has been suggested that it primarily channels glucose toward glycolysis [1, 7], whereas HK2 is mainly soluble and controls glycogen formation $[1,6,8]$. Distribution of HK2 between the cytosol and the mitochondria is dynamically regulated by glucose availability [6]. Increasing glucose uptake via the main glucose transporter GLUT4 $[9,10]$ mediates the interaction of HK2 with the outer mitochondrial membrane [11]. This interaction enhances the HK2's affinity to ATP $[2,7,12,13]$ and makes HK2 less sensitive to inhibition by its product G-6-P, which is further metabolized in the glycolytic pathway [12-14]. The translocation of HK2 to the mitochondria is facilitated by Akt-mediated phosphorylation of HK2 at the Thr473 residue [15] and this effect of Akt requires the presence of glucose [16]. Thus, both glucose and Akt signalling promote the binding of HK2 to the mitochondria, which may favor glucose catabolism over glycogen synthesis [6]. Akt also prevents HK2 dissociation from the mitochondria upon glucose removal and the ability of G-6-P to reduce the HK-mitochondria interaction [6].

Mitochondria-bound HKs highly support oxidative phosphorylation by increasing the availability of ADP for complex V of the respiratory chain [17] which helps to maintain a suitably low membrane potential and thus prevents the overproduction of the reactive oxygen species (ROS) [18]. HK bound to mitochondria via the voltage dependent anion channel (VDAC) [19-21] reduces the probability of apoptosis initiation [22-24] by inhibiting the binding of the proapoptotic protein Bax to the outer mitochondrial membrane [25], thereby preventing the mitochondrial permeability transition (MPT) pore opening and cytochrome $c$ release $[15,22,26]$. It has been shown that HK1 can also inhibit the formation of active proapoptotic caspases and block the mitochondrial step of tumor necrosis factor (TNF)mediated cell death [27]. These observations suggest that, besides its critical involvement in the regulation of glucose metabolism, HK can play a crucial role in protective signalling pathways. Indeed, the HK-Akt-mediated cardiomyocyte protection has been reported in some recent studies $[15,28,29]$. It has also been shown that the overexpression of full-length HK results in protection against cell death $[22,29,30]$.

Several reports have demonstrated significant right-to-left ventricular differences in myocardial susceptibility to various insults. For instance, the right ventricle (RV) seems to be less sensitive to anthracycline toxicity [31] and more resistant to ischemia-induced injury than the left ventricle (LV) $[32,33]$. However, the molecular mechanisms underlying these differences still remain unclear. In the present study, we found that the expression of both HK1 and HK2 is significantly higher in the RV than in the LV. In addition, we observed a higher Akt phosphorylation, which suggests an enhanced activation of the PI3K/Akt-HK pathway in the RV. These differences may contribute to a higher aerobic glycolytic metabolism of the RV and to its more resistant phenotype compared to the LV.

\section{Materials and Methods}

\section{Animals}

Adult male Wistar rats $(388 \pm 5.54 \mathrm{~g})$ were fed a standard laboratory diet and kept at a $12 / 12$-h light/dark cycle. The maintenance and handling of the experimental animals were in accordance with the Guide for the Care and Use of Laboratory Animals published by the US National Institutes of Health (NIH 
Publication No. 85-23, revised 1996). The experimental protocol was approved by the Animal Care and Use Committee of the Institute of Physiology, Academy of Sciences of the Czech Republic.

\section{Tissue preparation}

All rats were sacrificed by decapitation and the hearts were rapidly excised and washed in ice-cold saline. The LV and RV were dissected, immediately frozen in liquid nitrogen and weighed. Frozen tissue pieces were pulverized in liquid nitrogen and subsequently homogenized either in homogenization buffer (12.5 mM TRIS, $2.5 \mathrm{mM}$ EGTA, $1 \mathrm{mM}$ EDTA, $250 \mathrm{mM}$ sucrose, $5 \mathrm{mM}$ DTT, protease inhibitor cocktail (Complete, Roche Diagnostics), and phosphatase inhibitor cocktail (PhosSTOP, Roche Diagnostics), pH 7.4) for Western blotting (WB) and enzyme activities or in Trizol Reagent (Invitrogen) for isolation of mRNA. The homogenates for $\mathrm{WB}$ and enzyme activities were aliquoted and stored at $-80^{\circ} \mathrm{C}$. Protein concentration was measured using the Bradford dye binding assay [34].

\section{RNA isolation and Real-Time quantitative RT-PCR analysis}

The total cellular RNA was extracted from myocardial samples using TRIZOL Reagent (Invitrogen) and cleaned up by DNA-free RNA kit (Ambion). The purity and integrity of the RNA preparations was checked spectroscopically and by agarose gel electrophoresis. Ten $\mu$ g of total RNA was converted to cDNA using a RevertAidTM H Minus First Strand cDNA Synthesis Kit (Fermentas) with oligo(dT) primers according to the manufacturer's instructions. The primers for amplification of hexokinase isoform genes were designed using the Universal Probe Library Assay Design Center (Roche Applied Science). Sequences of the primers were: $H k 1$ (F): TCTGGGCTTCACCTTCTCAT and Hk1 (R): ATCAAGATTCCACAGTCCAGGT; $H k 2$ (F): CCAGCAGAACAGCCTAGACC and Hk2 (R): AGATGCCTTGAATCCCTTTG. Both pairs of primers were spanning introns. Each sample of cDNA was diluted 1:2 with RNAse free water and $1 \mu \mathrm{l}$ of this cDNA was then amplified in $10 \mu \mathrm{l}$ of PCR reaction mixture containing $1 \mu \mathrm{M}$ of HK isoform-specific primers and SyberGreen Master Mix (Bio-Rad) according to the manufacturer's instructions. Real-Time PCRs were performed on a LightCycler® 480 Real-Time PCR System (Roche Applied Science) using SyberGreen protocols. The data used for the calculations are the mean of the CT values obtained from qPCR performed in triplicates. The variation between triplicate determinations did not exceed $0.5 \mathrm{CT}$. Melting curve analysis was performed to ascertain the presence of a single amplicon for each pair of primers. Standard curves were generated for each pair of primers using a 3-fold serial dilution of cDNA. The amplification efficiency of the PCR reaction for each primer pair was then calculated from the standard curve in order to estimate precisely the relative transcript expression. Transcript levels were normalized to the level of the reference gene hypoxanthineguanine phosphoribosyl transferase 1 (HPRT) transcript. High expression stability of HPRT had been established previously [35]. The expression level of mRNA was normalized with regard to specific PCR efficiency (E) for each gene according to the following formula [36]:

$$
\text { Normalized amount }=(1+E)^{\mathrm{CT} \text { reference transcript }} /(1+\mathrm{E})^{\mathrm{CT} \text { target transcript }}
$$

No-template and no-RT control reactions were performed to screen for false amplification and to confirm the absence of DNA contamination.

\section{SDS-PAGE and Western blot analysis}

Individual homogenates from the LV and RV samples were separated by sodium dodecyl sulfate electrophoresis on $10 \%$ polyacrylamide gels at a constant voltage of $200 \mathrm{~V}$ (Mini-PROTEAN TetraCell, BioRad). The gel-resolved proteins were electrotransferred onto the nitrocellulose membrane $(0.2 \mu \mathrm{m}$ pore size, Protran BA 83, Whatman) at a constant voltage of $100 \mathrm{~V}$ and $350 \mathrm{~mA}$ current for $1 \mathrm{~h}$ (Mini Trans-Blot, Bio-Rad). Membranes were blocked for $1 \mathrm{~h}$ at room temperature with $5 \%$ non-fat dry milk in Tris-buffered saline solution containing Tween 20 (TTBS). After washing in TTBS, membranes were incubated overnight at $4{ }^{\circ} \mathrm{C}$ with the following primary polyclonal antibodies: rabbit anti-HK1, rabbit anti-HK2 (Santa Cruz Biotechnology), all rabbit anti-Akt, anti-phospho-Akt (Ser473), and anti-phospho-Akt (Thr308) (GenScript, Antibodies-online $\mathrm{GmbH}$ ). Next day, the membranes were washed in TTBS and incubated with anti-rabbit secondary antibody (GE Healthcare Amersham). Proteins were detected by enhanced chemiluminiscence (ECL) substrate (SuperSignal West Dura Extended Duration Substrate, Thermo Scientific Pierce) and visualized by a LAS-4000 imaging system (Genetica, Fujifilm). Changes in the relative protein levels were 
quantified using Quantity One Software (Bio-Rad). The same amount of protein (20 $\mu$ g per lane) was loaded on the gels. All samples from both ventricles were always run on the same gel and quantified on the same membrane. The analysis was repeated four times for each antibody. Glyceraldehyde 3-phosphate dehydrogenase (GAPDH) was used as a loading control to ensure that equal amount of protein was analyzed in each sample. Variation of the GAPDH immunoreactivity signal between the individual samples on each immunoblot was under $5 \%$ (data not shown).

\section{HK enzyme activity analysis}

Specific enzyme activity of HK was assessed by enzyme-coupled assays according to a slightly modified Worthington protocol (http://www.worthington-biochem.com/HK/; Worthington Biochemical Corporation). The assay buffer consisted of $0.05 \mathrm{M}$ TRIS, $13.3 \mathrm{mM} \mathrm{MgCl}, 0.8 \mathrm{mM}$ NAD, $0.8 \mathrm{mM}$ ATP, $0.5 \%$ Triton X-100, and $1 \mathrm{U} / \mathrm{ml} \mathrm{G-6-P}$ dehydrogenase (pH 8.0). Samples (60 $\mu \mathrm{g}$ per well) in $167 \mu \mathrm{l}$ of the HK assay buffer were placed into 96-well plates. The reaction was initiated after 2 min by the addition of $33 \mu$ of starting solution (1.5 M glucose in TRIS- $\mathrm{MgCl}_{2}$ buffer, $\mathrm{pH} \mathrm{8.0)}$ and the assay was run at $30^{\circ} \mathrm{C}$ for $15 \mathrm{~min}$. The measurement of absorbance at $339 \mathrm{~nm}$ wavelength was performed using a multireader Synergy ${ }^{\mathrm{TM}} \mathrm{HT}$ (Biotek Instruments). The HK specific enzyme activity was expressed as units per gram protein (U/g) [37].

\section{Immunofluorescence staining}

Ventricular myocardium for immunofluorescence staining was processed as described previously [38]. The subcellular localization of HK1 and HK2 and their co-localization with the mitochondrial membrane in the LV and RV were investigated by immunofluorescence staining of apex cross cryosections followed by fluorescence microscopy. Cryosections (5 to $7 \mu \mathrm{m}$ ) were fixed in $4 \%$ methanol-free formaldehyde (Polysciences, Inc.) and permeabilized in 100\% ice-cold methanol. Non-specific binding sites were blocked in an appropriate serum diluted in PBS containing 0.3\% Triton X-100. Cryosections were incubated with rabbit primary polyclonal antibodies against rat HK1 and HK2 (Santa Cruz Biotechnology) or, alternatively, with Alexa Fluor 488 conjugated rabbit monoclonal antibody against rat HK1 (Cell Signaling Technology). Sections stained with polyclonal sera were subsequently incubated with donkey anti-rabbit IgG secondary antibody conjugated with Alexa Fluor 488 (Invitrogen, Molecular Probes) or goat anti-rabbit IgG secondary antibody conjugated with Cy5 (KPL, Inc.). The mitochondrial compartment was stained with MitoProfile Total OXPHOS Rodent Antibody Cocktail (Abcam). Binding of primary antibodies was visualized with goat anti-mouse Alexa Fluor 647 secondary antibody (Invitrogen, Molecular Probes). Sections were mounted in ProLong Gold Antifade Reagent containing DAPI as a nuclei marker (Invitrogen, Molecular Probes).

\section{Quantitative fluorescence microscopy}

Representative qualitative images were captured by confocal microscopy (Leica TCS SP2). Images were acquired using a sequential scanning mode avoiding cross-talk between channels, 16 times line averaging, and noise reduction by 3 times frame averaging. For the purpose of quantitative analysis, images were acquired using a broad-band fluorescence microscope (Olympus Cell^R).

Myocardial expression of both HK isoforms was investigated by fluorescence intensity measurements using Fiji ImageJ open source software [39]. Eight positions (four for each ventricle) on every section were captured. A time-lapse scanning mode was used in order to prove the stability of fluorescence during the sample observation and acquisition. Each position was scanned 10 times and average intensity for each ventricle was calculated.

The correlation between the fluorescence signal of HK1 or HK2 isoforms and the mitochondrial OXPHOS complex was calculated using an ICA plugin of Fiji ImageJ software [40]. Eight positions on each cryosection (four for each ventricle) were sequentially acquired for a red (AlexaFluor 647) and green (AlexaFluor 488) channel. Each position was optically sectioned at $0.5 \mu \mathrm{m}$ steps resulting in approximately 12 focal planes depending on specimen thickness. Each position was captured twice to calculate the influence of the noise of an image. Regions of interest (ROI) were selected as sarcoplasmic myofibrilar regions excluding nucleus, perinuclear area and sarcolemma. The RBNCC method [41] was applied for the correction of noise when calculating the Pearson's correlation coefficients between the green channel representing HK1 or HK2 and the red channel representing OXPHOS complexes. 
Fig. 1. Real-Time RT-PCR and Western blot analysis of HK1 (A, C) and HK2 (B, D) expression in the LV and RV. The amount of protein applied on the gel was $20 \mu \mathrm{g}$. The values of mRNA are normalized to the reference gene hypoxanthine phosphoribosyltransferase (HPRT). Specific enzyme activity of HK (E) is expressed in $\mathrm{U} / \mathrm{g}$ of protein. Data are expressed as percentages of total immunoreactivity or relative mRNA expression of HK determined in both $\mathrm{LV}$ and RV (100\%). Values are mean \pm SEM from 10 hearts. ${ }^{*} p<0.05$ vs LV.

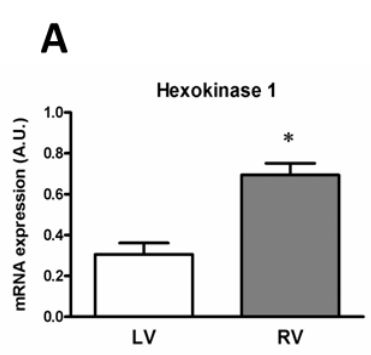

\section{B}

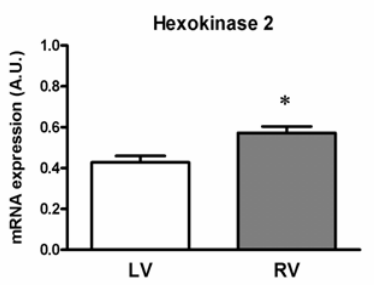

C

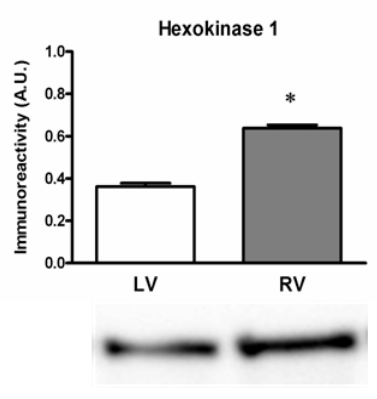

D

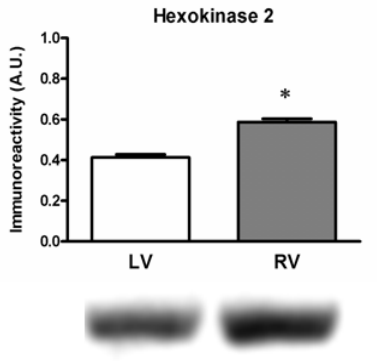

$\mathrm{E}$

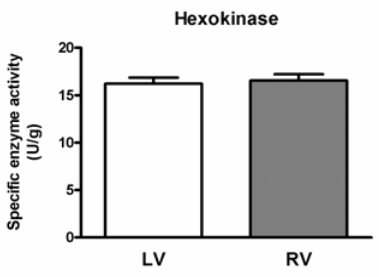

\section{Statistical analysis}

Samples of 10 rat hearts were used for WB and Real-Time RT-PCR analyses and 6 hearts were used for quantitative fluorescence analyses. All data are expressed as mean \pm SEM. The statistical differences between the ventricles were determined by the unpaired Mann-Whitney test $(p<0.05$ or $p<0.001)$. Percentages of total immunoreactivity, relative mRNA expression or fluorescence intensity of HK and immunoreactivity of phosphorylated Akt were determined in both the LV and RV (100\%).

\section{Results}

\section{Hexokinase expression and enzyme activity}

The Real-Time RT-PCR analyses (Fig. 1A, B) showed that the mRNA levels of both HK1 and HK2 were higher by $128 \%$ and $34 \%$, respectively, in the RV than in the LV. The protein levels of HK1 and HK2 followed this pattern: they were also markedly higher (by $76 \%$ and $42 \%$, respectively) in the RV than in the LV (Fig. 1C, D). The hexokinase specific enzyme activity did not significantly differ between the ventricles (Fig. 1E).

Quantitative immunofluorescence microscopy analysis of HK distribution

Expression of both HK1 and HK2 isoforms in the RV was also verified by immunofluorescence staining of the cross cryosections. HK1 as well as HK2 manifested significantly higher fluorescence intensity (by $24 \%$ and $22 \%$, respectively) in the RV 


\section{A}
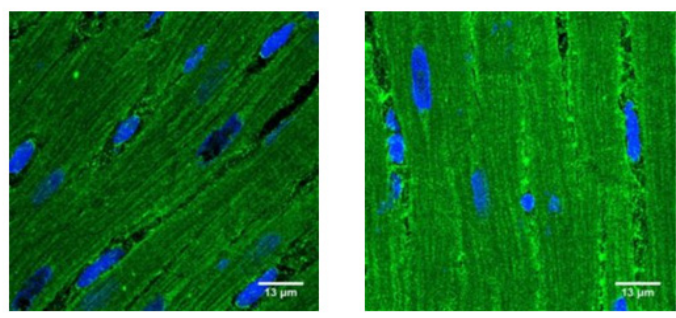

C
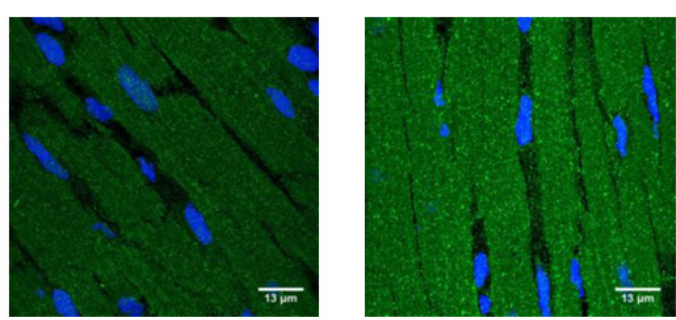

B

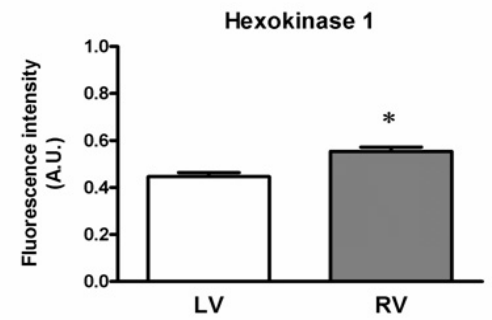

D

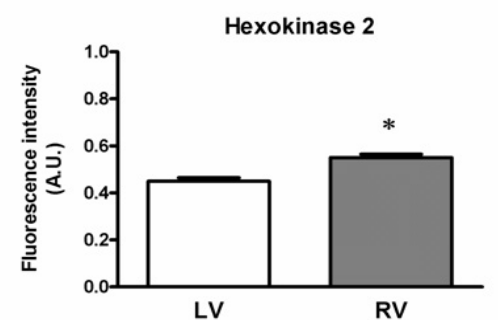

Fig. 2. Representative micrographs showing the distribution of HK1 (A) and HK2 (C) in the LV and RV (the left and right micrographs, respectively) vizualized in cross cryosections of rat hearts using a fluorescence microscope. The green color corresponds to specific HK staining and the blue color indicates the nuclear 4',6-diamidino-2-phenylindole (DAPI) staining. Scale bar represents $13 \mu \mathrm{m}$. Quantification of the mean intensity of fluorescence of HK1 (B) and HK2 (D) in the LV and RV are expressed as a percentage of the total intensity of fluorescence signals determined in both LV and RV (100\%). Values are mean \pm SEM from 6 hearts. ${ }^{*} p<0.001$ vs LV.

Fig. 3. Determination of phosporylated Akt in the $\mathrm{LV}$ and RV. Western blot analyses of phosphorylated Akt at Ser473 residue (pS-Akt) and Thr308 residue (pT-Akt) were conducted using specific antibodies. Results of quantitative densitometric analyses are expressed as percentages of the total immunoreactivity of pS-Akt (A) and pT-Akt (B) determined in both LV and RV (100\%) and as a ratio of phosphorylated-to-nonphopshorylated Akt: pS-Akt/ Akt (C) and pT-Akt/Akt (D). The amount of total protein applied on the gel was $20 \mu$ g. Values are mean \pm SEM from 10 hearts. ${ }^{*} p<0.05 v s$ LV.
A

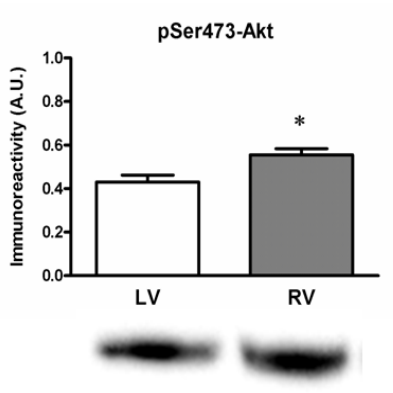

C

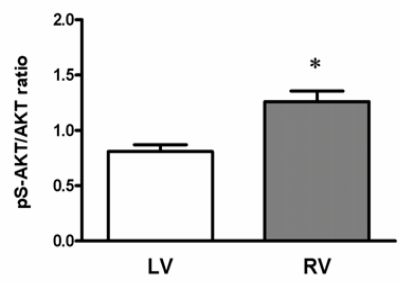

B

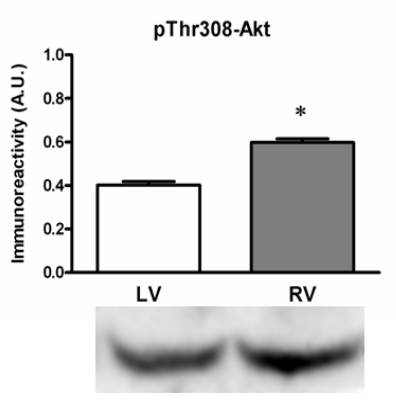

D

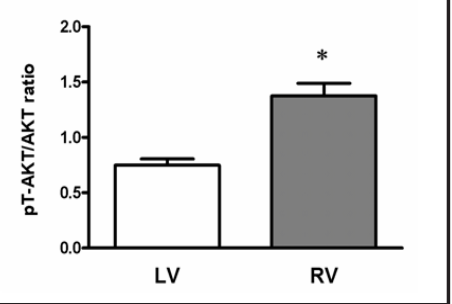

compared to the LV (Fig. 2A-D). Similar results were obtained by using another primary antibody (monoclonal antibody conjugated with Alexa 488) and different secondary antibody conjugated with Cy5 fluorophore (data not shown). 


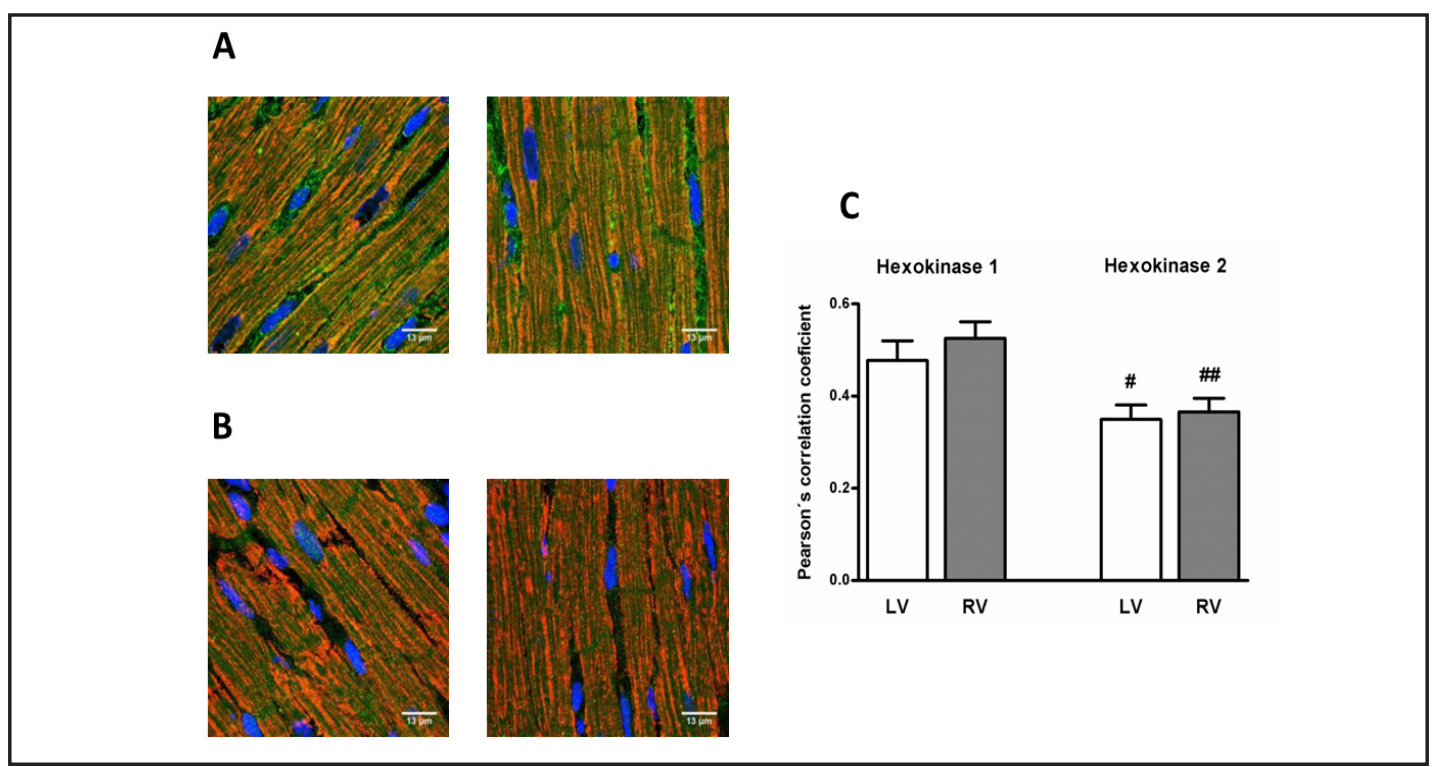

Fig. 4. Representative micrographs showing distribution and co-localization of HK1 and HK2 with mitochondria in cross cryosections of the LV and RV obtained by a confocal microscope. In all micrographs, green color represents specific HK1 (A) or HK2 (B) staining and blue color indicates the nuclear 4',6-diamidino-2-phenylindole (DAPI) staining. Red color represents the distribution of the OXPHOS complexes. The increase in yellow-orange color in both panels indicates an increased co-localization of HK1 or HK2 with mitochondria in both LV and RV (the left and right micrographs, respectively). Scale bar represents $13 \mu \mathrm{m}$. Quantification of co-localization of HK1 and HK2 with mitochondria (OXPHOS complexes) using the Pearson's correlation coefficients in cryosections from the LV and RV (C). Values are mean \pm SEM from 6 hearts. ${ }^{\#}<0.05$ vs HK1 LV, ${ }^{\# \#} p<0.05$ vs HK1 RV.

\section{Western blot analysis of Akt}

The phospho-Ser473-Akt as well as the phospho-Thr308-Akt levels were significantly higher in the RV than in the LV (Fig. 3A, B), similarly as the ratio of phosphorylated to nonphosphorylated Akt (Fig. 3C, D), indicating a higher activation of Akt in the RV, which is required for the interaction of HK2 with mitochondria.

\section{Subcellular localization of $\mathrm{HK}$ and co-localization of $\mathrm{HK}$ with mitochondria}

It has been previously demonstrated that the higher phosphorylation of Akt is usually associated with higher co-localization of HKs with mitochondria [15]. As can be seen in Fig. 4A and 4B, the HK isoforms displayed different patterns of fluorescence signals in cardiomyocytes. In contrast to a fuzzy pattern of HK2 distribution, HK1 appeared to be organized rather into longitudinal arrays or chains. Pearson's correlation coefficients between the green (HK1 or HK2) and the red channels (OXPHOS mitochondria) were calculated to further quantify differences in the co-localization of HKs with the mitochondria. Neither co-localization of HK1 (0.48 \pm 0.04 vs $0.53 \pm 0.04)$ nor co-localization of HK2 (0.35 \pm 0.03 vs $0.37 \pm 0.03$ ) with mitochondria differed between the LV and the RV. However, the co-localization of HK2 with mitochondria was significantly lower compared to HK1 in both ventricles (Fig. 4C).

\section{Discussion}

In the present study, we demonstrated a significantly higher expression of HK1 and HK2 isoforms at mRNA, as well as protein level in the RV as compared to the LV, although the total HK activity did not differ between the ventricles. These findings were confirmed 
by immunofluorescence staining and fluorescence microscopy analysis, which revealed substantially higher fluorescence signals of both HKs in the RV than in the LV. Moreover, we found a higher phosphorylation of Akt at the Ser473 residue as well as the Thr308 residue in the RV.

The LV and RV exhibit striking differences in anatomical and functional characteristics such as the size, shape, wall thickness, myofiber architecture, as well as systolic and diastolic properties, reflecting their diverse roles in the circulation and determining their different responses to various loading conditions and disease states [42-45]. Concerning myocardial energetics, the oxygen requirements of the $\mathrm{RV}$ is lower compared to the $\mathrm{LV}$ due to lower wall stress, resulting in a larger oxygen supply reserve [45] making the RV less vulnerable to conditions associated with increased energy demands. In view of these differences, it seems conceivable that the RV and LV may differ in the expression profiles of genes and proteins controlling myocardial energy metabolism. However, so far there are only a few studies addressing the metabolic differences between the RV and LV on the molecular level $[46,47]$.

Most studies dealing with HK in the heart focused only on the enzyme activity in homogenate. Myocardial HK activity differs among species and exhibits the transmural gradient $[48,49]$. It was reported that HK exhibits the maximal enzyme activity in the heart during the fetal period and only moderate changes occur during postnatal life [50,51]. Although higher activity of HK in the RV compared to the LV was found in young rats [49, 51], other studies did not detect any significant right-to-left ventricular difference [52-54] according to results of the present work. To our knowledge, no data have been published to date comparing the expression and subcellular localization of HK isoforms in the RV and LV. The higher content of HK in the RV demonstrated in the present study may suggest a higher activity of aerobic glycolytic metabolism in this ventricle, which can enhance oxidative phosphorylation and consequently attenuate oxidative stress. This notion is supported by recent findings of the higher expression of insulin-like growth factor 1 (IGF-1) in the RV than in the LV [46]. IGF-1 is an important regulator of glucose uptake and utilization [55]. Besides that the RV shows a lower expression of both fatty acid binding protein isoforms and a higher expression of glycogen debranching enzyme [47] which may favor higher glucose utilization in the RV. A higher activity of glucose metabolism is associated with a faster contraction related to myosin heavy chain (MyHC) isoform expression. Interestingly, a lower expression of MyHC $\beta$ and a higher expression of $\mathrm{MyHC} \alpha$ with higher ATPase activity [56-58] in the $\mathrm{RV}$ as compared to the $\operatorname{LV}[59,60]$, indicate that the RV exhibits a faster muscle phenotype. Indeed, the shortening velocity of the RV muscle is greater than that of the LV [59].

Some experimental studies demonstrated that the RV is more resistant than the LV to the toxic effect of anthracycline and to the injury caused by acute ischemia [31-33], suggesting that more efficient protective mechanism(s) may operate in the RV. However, one cannot eliminate whether the better ischemic tolerance of the RV is due to its lower workload and energy demands. Interestingly, several reports showed that an increased expression of $\mathrm{HK}$ is associated with an improved resistance of cells, including cardiomyocytes, against ischemic injury [61] and apoptosis [15, 22, 25, 28-30]. HK is a crucial glycolytic enzyme playing an important role in survival pathways. Both HK isoforms are essential for the normal physiological function of the heart and are not mutually substitutable. The depletion of the HK1 isoform results in caspase-8-dependent cell death in response to TNF [27], which points out to a strong pro-survival function of this enzyme. The physiological importance of HK1 probably lies to a high degree in the association of this isoform with mitochondria, because its function in glycolysis could be replaced by HK2. Accordingly, the binding of HK2 to mitochondria has been shown to be necessary for the normal function of mitochondria. A recent study demonstrated that the displacement of HK2 from isolated mitochondria using an artificial peptide resulted in the enhanced release of cytochrome $c$ upon treatment with the recombinant tBid, a membrane-targeted death ligand [62]. In addition, the disruption of HK2 binding to mitochondria blocks ischemic preconditioning and may cause myocardial necrosis [63] or apoptosis [28]. 
The importance of the association of HK with mitochondria for the regulation of its activity was proved by experiments demonstrating that the enzyme activity doubled during the first hour after HK translocation to the mitochondria and continued to rise thereafter [64]. However, in the present study we did not find any difference in total HK activity or in its association with mitochondria between the ventricles despite the higher expression of HK1 and HK2 in the RV as compared to the LV. This apparent discordance may be explained by the fact that comparable amounts of both HK isoforms bound to mitochondria were detected in both ventricles. The increased total amount of HK in the RV as compared to the LV can be due to higher levels of cytosolic forms of the enzyme. Taking into account that HK associated with mitochondria contributes principally to a higher enzyme activity, thus the increased levels of cytosolic HK in the RV need not substantially affect the total enzyme activity. Moreover, changes in the total HK activity correlate with specific changes in the activity of the cytosolic isoform HK2 rather than with the activity of HK1, which is predominantly bound to mitochondria [65].

When comparing the expression and activity of HK, it is important to keep in mind that standard Western blot analysis under denaturing conditions only allows the detection of the monomeric form of the enzyme, but the dimers or tetramers may substantially differ in their activity [66]. Posttranslational modifications such as sumoylation [67] and ubiquitinylation [68] can affect the enzyme activity of HK and cause its degradation. The majority of soluble HK2 in brain tissue has been found to be ubiquitinylated at the N-terminal portion of the molecule, which may increase its susceptibility to degradation [68] and affect its enzyme activity [24]. Hence, the enzyme activity need not be directly proportional to the relative amount of HK determined by Western blots. Moreover, tissue homogenization could disrupt many of these levels of regulation, including detaching HK from mitochondria. It means that in vitro measurements of HK activity may differ from the real in vivo tissue activities.

The mechanisms underlying the enhancement of enzyme activity induced by HK interaction with the mitochondria include conformational changes of the enzyme molecule and Pi competition with G-6-P at its binding site. HK2 forms dimers in the cytosol while the interaction of HK2 with mitochondria requires HK in a tetrameric form, as well as the HK1mitochondria interaction $[69,70]$. The HK monomer-dimer-tetramer transition and their interactions with mitochondria can further affect the conformational state of the monomers and thus also change their substrate-binding affinity resulting in the increased activity of the whole complex. The question then arises how oligomerization of HK2 can change the enzyme activity of monomers. Another question is whether the increased enzyme activity due to it binding to the mitochondria is caused by product channeling [71], i.e. by a shift of the equilibrium to the right, or through an increased number of active monomers formed by conformational changes induced by the interaction itself. Monomers of HK1 and HK2 share a high degree of functional similarity between certain domains but there are some differences. The first 15 hydrophobic N-terminal amino acid residues are strictly required for association of HK1 as well as HK2 with VDAC on the outer side of the mitochondrial membrane [7275]. The N-terminal part of both isoforms also possesses a regulatory domain containing G-6-P and latent glucose binding sites. An active site of both isoforms is located at the central C-terminal part of each monomer and, additionally, the N-terminal part of HK2 also exhibits catalytic properties [24]. A strong regulatory effect of Pi is based on the direct competitive inhibitory mechanism attenuating G-6-P binding to the N-terminal half of the molecule at physiological concentrations [72, 76-78] and an indirect displacement of G-6-P from the catalytic site (C-terminal half) at HK1 [79]. HK2 is more sensitive to the inhibition by its G-6-P product, because it is not antagonized by Pi at the N-terminal domain [80]. Importantly, HK binding to the mitochondria may also protect the enzyme against degradation. It has been demonstrated that the soluble, but not the mitochondria-bound, hexokinase becomes a substrate for the ATP- and ubiquitin-dependent proteolytic system [68].

Our immunofluorescence analysis showed that the association of HK1 with mitochondria is greater than with the association of HK2 in both ventricles. Southworth et al. [11] performed a very detailed study focused on the distribution of HK in LV cardiomyocytes using electron 
microscopy. They found that in the resting heart, HK1 is associated with mitochondria to a much higher extent (10-fold) than HK2 and that the binding of each enzyme isoform to mitochondria is regulated differently. Interestingly, under ischemic conditions or increased level of insulin [11] as well as under increased concentration of glucose [6], the association of HK2 with mitochondria increased 5-fold, while that of HK1 only 2-fold [11].

Recently, it has been reported that fully activated phospho-Akt stimulates HK2 translocation to the outer mitochondrial membrane [15] where HK2 inhibits the binding of the Bax protein [25] and the opening of the MPT pore [22,26]. Full activation of Akt requires phosphorylation at both Ser and Thr residues and it has been demonstrated in HEK293 cells that the phosphorylation of Thr308 and Ser473 is independent of each other [81]. The Ser473 residue can be also phosphorylated by MAPKAP-K2, the activation of which is not dependent on the PI3-kinase pathway [82]. In the present study, we found that the expression of phospho-Ser-Akt as well as phospho-Thr-Akt is significantly higher in the RV than in the LV. In addition, both ratios of phospho-Ser-Akt/non-phosphorylated Akt and phospho-ThrAkt/non-phosphorylated Akt were markedly higher in the RV than in the LV. Nevertheless, the higher level of activated Akt in the RV did not lead to an increased association of HK2 with the mitochondria. These findings suggest that Akt activation is a necessary but not a sufficient condition for the enhancement of the interaction of HK2 with mitochondria and that yet another mechanism may exist, initiated by a complex physiological processes such as an increased concentration of glucose or insulin, for which the increased binding of HK2 to mitochondria was described $[6,11]$.

In conclusion, our present study has revealed significantly higher amounts of HK1 and HK2 and increased phosphorylation of Akt in the RV as compared to the LV. These results suggest that the RV has a higher activity of aerobic glycolytic metabolism and may be able to respond faster and more powerfully to stressful stimuli than the LV.

\section{Disclosure}

The authors declare that they have no competing interests.

\section{Acknowledgements}

This work was supported by the Charles University Grant Agency (19010), the Ministry of Education of the Czech Republic (MSM0021620858), the Grant Agency of the Academy of Science of the Czech Republic (IAAX01110901 and IAA601110908) and by the grant SVV2012-265206.

\section{References}

1 Wilson JE: Isozymes of mammalian hexokinase: structure, subcellular localization and metabolic function. J Exp Biol 2003;206:2049-2057.

-2 Aubert-Foucher E, Font B, Gautheron DC: Rabbit heart mitochondrial hexokinase: solubilization and general properties. Arch Biochem Biophys 1984;232:391-399.

-3 Burcelin R, Printz RL, Kande J, Assan R, Granner DK, Girard J: Regulation of glucose transporter and hexokinase II expression in tissues of diabetic rats. Am J Physiol 1993;265:E392-401.

4 White TK, Wilson JE: Binding of nucleoside triphosphates, inorganic phosphate, and other polyanionic ligands to the N-terminal region of rat brain hexokinase: relationship to regulation of hexokinase activity by antagonistic interactions between glucose 6-phosphate and inorganic phosphate. Arch Biochem Biophys 1990;277:26-34. 
Waskova-Arnostova/Elsnicova/Kasparova et al.: Hexokinase and Akt in Rat Heart

5 Mathupala SP, Ko YH, Pedersen PL: Hexokinase-2 bound to mitochondria: cancer's stygian link to the "Warburg Effect" and a pivotal target for effective therapy. Semin Cancer Biol 2009;19:17-24.

6 John S, Weiss JN, Ribalet B: Subcellular localization of hexokinases I and II directs the metabolic fate of glucose. PLoS ONE 2011;6:e17674.

7 Wilson JE: An introduction to the isoenzymes of mammalian hexokinase types I-III. Biochem Soc Trans 1997;25:103-107.

8 Sebastian S, Horton JD, Wilson JE: Anabolic function of the type II isozyme of hexokinase in hepatic lipid synthesis. Biochem Biophys Res Commun 2000;270:886-891.

-9 Abel ED: Glucose transport in the heart. Front Biosci 2004;9:201-215.

10 Postic C, Leturque A, Printz RL, Maulard P, Loizeau M, Granner DK, Girard J: Development and regulation of glucose transporter and hexokinase expression in rat. Am J Physiol 1994;266:E548-559.

11 Southworth R, Davey KAB, Warley A, Garlick PB: A reevaluation of the roles of hexokinase I and II in the heart. Am J Physiol Heart Circ Physiol 2007;292:H378-386.

12 Bustamante E, Pedersen PL: High aerobic glycolysis of rat hepatoma cells in culture: role of mitochondrial hexokinase. Proc Natl Acad Sci USA 1977;74:3735-3739.

13 Depré C, Rider MH, Hue L: Mechanisms of control of heart glycolysis. Eur J Biochem 1998;258:277-290.

14 Bustamante E, Morris HP, Pedersen PL: Energy metabolism of tumor cells. Requirement for a form of hexokinase with a propensity for mitochondrial binding. J Biol Chem 1981 25;256:8699-8704.

15 Miyamoto S, Murphy AN, Brown JH: Akt mediates mitochondrial protection in cardiomyocytes through phosphorylation of mitochondrial hexokinase-II. Cell Death Differ 2008;15:521-529.

16 Majewski N, Nogueira V, Robey RB, Hay N: Akt inhibits apoptosis downstream of BID cleavage via a glucose-dependent mechanism involving mitochondrial hexokinases. Mol Cell Biol 2004;24:730-740.

17 Gottlob K, Majewski N, Kennedy S, Kandel E, Robey RB, Hay N: Inhibition of early apoptotic events by Akt/ PKB is dependent on the first committed step of glycolysis and mitochondrial hexokinase. Genes Dev 2001;15:1406-1418.

18 da-Silva WS, Gómez-Puyou A, de Gómez-Puyou MT, Moreno-Sanchez R, De Felice FG, de Meis L, Oliveira MF, Galina A: Mitochondrial bound hexokinase activity as a preventive antioxidant defense: steady-state ADP formation as a regulatory mechanism of membrane potential and reactive oxygen species generation in mitochondria. J Biol Chem 2004;279:39846-39855.

19 Anflous-Pharayra K, Cai Z-J, Craigen WJ: VDAC1 serves as a mitochondrial binding site for hexokinase in oxidative muscles. Biochim Biophys Acta 2007;1767:136-142.

20 Rosano C: Molecular model of hexokinase binding to the outer mitochondrial membrane porin (VDAC1): Implication for the design of new cancer therapies. Mitochondrion 2011;11:513-519.

21 Shoshan-Barmatz V, Zakar M, Rosenthal K, Abu-Hamad S: Key regions of VDAC1 functioning in apoptosis induction and regulation by hexokinase. Biochim Biophys Acta 2009;1787:421-430.

-22 Azoulay-Zohar H, Israelson A, Abu-Hamad S, Shoshan-Barmatz V: In self-defence: hexokinase promotes voltage-dependent anion channel closure and prevents mitochondria-mediated apoptotic cell death. Biochem J 2004;377:347-355.

23 Majewski N, Nogueira V, Bhaskar P, Coy PE, Skeen JE, Gottlob K, Chandel NS, Thompson CB, Robey RB, Hay $\mathrm{N}$ : Hexokinase-mitochondria interaction mediated by Akt is required to inhibit apoptosis in the presence or absence of Bax and Bak. Mol Cell 2004;16:819-830.

24 Pastorino JG, Hoek JB: Hexokinase II: the integration of energy metabolism and control of apoptosis. Curr Med Chem 2003;10:1535-1551.

25 Pastorino JG, Shulga N, Hoek JB: Mitochondrial binding of hexokinase II inhibits Bax-induced cytochrome c release and apoptosis. J Biol Chem 2002;277:7610-7618.

-26 Beutner G, Rück A, Riede B, Brdiczka D: Complexes between porin, hexokinase, mitochondrial creatine kinase and adenylate translocator display properties of the permeability transition pore. Implication for regulation of permeability transition by the kinases. Biochim Biophys Acta 1998;1368:7-18.

-27 Schindler A, Foley E: A functional RNAi screen identifies hexokinase 1 as a modifier of type II apoptosis. Cell Signal 2010;22:1330-1340.

-28 Chiara F, Castellaro D, Marin O, Petronilli V, Brusilow WS, Juhaszova M, Sollott SJ, Forte M, Bernardi P, Rasola A: Hexokinase II detachment from mitochondria triggers apoptosis through the permeability transition pore independent of voltage-dependent anion channels. PLoS ONE 2008;3:e1852. 
Waskova-Arnostova/Elsnicova/Kasparova et al.: Hexokinase and Akt in Rat Heart

29 Sun L, Shukair S, Naik TJ, Moazed F, Ardehali H: Glucose phosphorylation and mitochondrial binding are required for the protective effects of hexokinases I and II. Mol Cell Biol 2008;28:1007-1017.

-30 Ahmad A, Ahmad S, Schneider BK, Allen CB, Chang L-Y, White CW: Elevated expression of hexokinase II protects human lung epithelial-like A549 cells against oxidative injury. Am J Physiol Lung Cell Mol Physiol 2002;283:L573-584.

-31 Belham M, Kruger A, Pritchard C: The Tei index identifies a differential effect on left and right ventricular function with low-dose anthracycline chemotherapy. J Am Soc Echocardiogr 2006;19:206-210.

-32 Baker JE, Holman P, Kalyanaraman B, Griffith OW, Pritchard KA Jr: Adaptation to chronic hypoxia confers tolerance to subsequent myocardial ischemia by increased nitric oxide production. Ann N Y Acad Sci 1999;874:236-253.

33 Dell'Italia LJ: The right ventricle: anatomy, physiology, and clinical importance. Curr Probl Cardiol 1991;16:653-720.

34 Bradford MM: A rapid and sensitive method for the quantitation of microgram quantities of protein utilizing the principle of protein-dye binding. Anal Biochem 1976;72:248-254.

-35 Bohuslavova R, Kolar F, Kuthanova L, Neckar J, Tichopad A, Pavlinkova G: Gene expression profiling of sex differences in HIF1-dependent adaptive cardiac responses to chronic hypoxia. J Appl Physiol 2010;109:1195-1202.

-36 Pfaffl MW: A new mathematical model for relative quantification in real-time RT-PCR. Nucleic Acids Res 2001;29:e45.

37 Units of Enzyme Activity. European Journal of Biochemistry 1979;97:319-320.

-38 Hlavackova M, Kozichova K, Neckar J, Kolar F, Musters RJP, Novak F, Novakova O: Up-regulation and redistribution of protein kinase C- $\delta$ in chronically hypoxic heart. Mol Cell Biochem 2010;345:271-282.

-39 Schindelin J, Arganda-Carreras I, Frise E, Kaynig V, Longair M, Pietzsch T, Preibisch S, Rueden C, Saalfeld S, Schmid B, Tinevez JY, White DJ, Hartenstein V, Eliceiri K, Tomancak P, Cardona A: Fiji: an open-source platform for biological-image analysis. Nat Methods 2012;9:676-682.

40 Li Q Lau A, Morris TJ, Guo L, Fordyce CB, Stanley EF: A syntaxin 1, Galpha(o), and N-type calcium channel complex at a presynaptic nerve terminal: analysis by quantitative immunocolocalization. J Neurosci 2004;24:4070-4081.

41 Adler J, Pagakis SN, Parmryd I: Replicate-based noise corrected correlation for accurate measurements of colocalization. J Microsc 2008;230:121-133.

42 Haddad F, Hunt SA, Rosenthal DN, Murphy DJ: Right ventricular function in cardiovascular disease, part I: Anatomy, physiology, aging, and functional assessment of the right ventricle. Circulation 2008;117:14361448.

43 Ho SY, Nihoyannopoulos P: Anatomy, echocardiography, and normal right ventricular dimensions. Heart 2006;92:i2-i13.

44 Lorenz CH, Walker ES, Morgan VL, Klein SS, Graham TP Jr: Normal human right and left ventricular mass, systolic function, and gender differences by cine magnetic resonance imaging. J Cardiovasc Magn Reson 1999;1:7-21.

45 Walker LA, Buttrick PM: The right ventricle: biologic insights and response to disease. Curr Cardiol Rev 2009;5:22-28.

46 Drake JI, Bogaard HJ, Mizuno S, Clifton B, Xie B, Gao Y, Dumur CI, Fawcett P, Voelkel NF, Natarajan R: Molecular signature of a right heart failure program in chronic severe pulmonary hypertension. Am J Respir Cell Mol Biol 2011;45:1239-1247.

-47 Phillips D, Aponte AM, Covian R, Neufeld E, Yu Z-X, Balaban RS: Homogenous protein programming in the mammalian left and right ventricle free walls. Physiol Genomics 2011;43:1198-1206.

48 De Tata V, Fierabracci V, Gori Z, Bergamini E: Transmural distribution of glucose metabolizing enzymes across the left and the right ventricle heart walls in three different mammalian species. Comp Biochem Physiol, B 1986;84:549-553.

49 De Tata V, Gori Z, Bergamini E: Changes in the transmural distribution of glucose-metabolizing enzymes across the left and right ventricular wall of rat heart during growth and ageing. Arch Gerontol Geriatr 1988; 7:23-30.

50 Andrés A, Satrústegui J, Machado A: Development of enzymes of energy metabolism in rat heart. Biol Neonate 1984;45:78-85. 
Waskova-Arnostova/Elsnicova/Kasparova et al.: Hexokinase and Akt in Rat Heart

51 Bass A, Stejskalova M, Stieglerova A, Ostadal B, Samanek M: Ontogenetic development of energy-supplying enzymes in rat and guinea-pig heart. Physiol Res 2001;50:237-245.

52 Bass A, Stejskalova M, Ostadal B, Samanek M: Differences between atrial and ventricular energy-supplying enzymes in five mammalian species. Physiol Res 1993;42:1-6.

-53 Rumsey WL, Abbott B, Bertelsen D, Mallamaci M, Hagan K, Nelson D, Erecinska M: Adaptation to hypoxia alters energy metabolism in rat heart. Am J Physiol 1999;276:H71-80.

54 Daneshrad Z, Garcia-Riera MP, Verdys M, Rossi A: Differential responses to chronic hypoxia and dietary restriction of aerobic capacity and enzyme levels in the rat myocardium. Mol Cell Biochem 2000;210:159166.

55 Bondy CA, Cheng CM: Signaling by insulin-like growth factor 1 in brain. Eur J Pharmacol 2004;490:25-31.

-56 Ebrecht G, Rupp H, Jacob R: Alterations of mechanical parameters in chemically skinned preparations of rat myocardium as a function of isoenzyme pattern of myosin. Basic Res Cardiol 1982;77:220-234.

57 Pope B, Hoh JF, Weeds A: The ATPase activities of rat cardiac myosin isoenzymes. FEBS Lett 1980;118:205208.

58 Schwartz K, Lompre AM, Bouveret P, Wisnewsky C, Whalen RG: Comparisons of rat cardiac myosins at fetal stages in young animals and in hypothyroid adults. J Biol Chem 1982;257:14412-14418.

59 Brooks WW, Bing OH, Blaustein AS, Allen PD: Comparison of contractile state and myosin isozymes of rat right and left ventricular myocardium. J Mol Cell Cardiol 1987;19:433-440.

60 Sharma S, Razeghi P, Shakir A, Keneson BJ 2nd, Clubb F, Taegtmeyer H: Regional heterogeneity in gene expression profiles: a transcript analysis in human and rat heart. Cardiology 2003;100:73-79.

61 Zuurbier CJ, Smeele KMA, Eerbeek O: Mitochondrial hexokinase and cardioprotection of the intact heart. J Bioenerg Biomembr 2009;41:181-185.

62 Shulga N, Wilson-Smith R, Pastorino JG: Hexokinase II detachment from the mitochondria potentiates cisplatin induced cytotoxicity through a caspase-2 dependent mechanism. Cell Cycle 2009;8:3355-3364.

63 Smeele KMA, Southworth R, Wu R, Xie C, Nederlof R, Warley A, Nelson JK, van Horssen P, van den Wijngaard JP, Heikkinen S, Laakso M, Koeman A, Siebes M, Eerbeek O, Akar FG, Ardehali H, Hollmann MW, Zuurbier CJ: Disruption of hexokinase II-mitochondrial binding blocks ischemic preconditioning and causes rapid cardiac necrosis. Circ Res 2011;108:1165-1169.

64 Parra J, Brdiczka D, Cusso R, Pette D: Enhanced catalytic activity of hexokinase by work-induced mitochondrial binding in fast-twitch muscle of rat. FEBS Lett 1997;403:279-282.

65 Riddle SR, Ahmad A, Ahmad S, Deeb SS, Malkki M, Schneider BK, Allen CB, White CW: Hypoxia induces hexokinase II gene expression in human lung cell line A549. Am J Physiol Lung Cell Mol Physiol 2000;278:L407-416.

66 Hoggett JG, Kellett GL: Kinetics of the monomer-dimer reaction of yeast hexokinase PI. Biochem J 1992;287:567-572.

67 Aslanukov A, Bhowmick R, Guruju M, Oswald J, Raz D, Bush RA, Sieving PA, Lu X, Bock CB, Ferreira PA: RanBP2 modulates Cox11 and hexokinase I activities and haploinsufficiency of RanBP2 causes deficits in glucose metabolism. PLoS Genet 2006;2:e177.

68 Magnani M, Crinelli R, Antonelli A, Casabianca A, Serafini G: The soluble but not mitochondrially bound hexokinase is a substrate for the ATP- and ubiquitin-dependent proteolytic system. Biochim Biophys Acta 1994;1206:180-190.

69 Mulichak AM, Wilson JE, Padmanabhan K, Garavito RM: The structure of mammalian hexokinase-1. Nat Struct Biol 1998;5:555-560.

70 Wilson JE: Hexokinases. Rev Physiol Biochem Pharmacol 1995;126:65-198.

71 Gregor M, Janovska A, Stefl B, Zurmanova J, Mejsnar J: Substrate channelling in a creatine kinase system of rat skeletal muscle under various pH conditions. Exp Physiol 2003;88:1-6.

72 Rose IA, Warms JV: Mitochondrial hexokinase. Release, rebinding, and location. J Biol Chem 1967;242:1635-1645.

73 Lindén M, Gellerfors P, Nelson BD: Pore protein and the hexokinase-binding protein from the outer membrane of rat liver mitochondria are identical. FEBS Lett 1982;141:189-192.

-74 Fiek C, Benz R, Roos N, Brdiczka D: Evidence for identity between the hexokinase-binding protein and the mitochondrial porin in the outer membrane of rat liver mitochondria. Biochim Biophys Acta 1982;688:429-440. 
75 Kurokawa M, Oda S, Tsubotani E, Fujiwara H, Yokoyama K, Ishibashi S: Characterization of hexokinase isoenzyme types I and II in ascites tumor cells by an interaction with mitochondrial membrane. Mol Cell Biochem 1982;45:151-157.

76 Ellison WR, Lueck JD, Fromm HJ: Studies on the kinetics and mechanism of orthophosphate activation of bovine brain hexokinase. Biochem Biophys Res Commun 1974;57:1214-1220.

77 Ellison WR, Lueck JD, Fromm HJ: Studies on the mechanism of orthophosphate regulation of bovine brain hexokinase. J Biol Chem 1975;250:1864-1871.

-78 Tsai HJ, Wilson JE: Functional organization of mammalian hexokinases: characterization of chimeric hexokinases constructed from the $\mathrm{N}$ - and C-terminal domains of the rat type I and type II isozymes. Arch Biochem Biophys 1995;316:206-214.

79 Fang TY, Alechina O, Aleshin AE, Fromm HJ, Honzatko RB: Identification of a phosphate regulatory site and a low affinity binding site for glucose 6-phosphate in the N-terminal half of human brain hexokinase. J Biol Chem 1998;273:19548-19553.

80 Lueck JD, Fromm HJ: Kinetics, mechanism, and regulation of rat skeletal muscle hexokinase. J Biol Chem 1974;249:1341-1347.

81 Alessi DR, Andjelkovic M, Caudwell B, Cron P, Morrice N, Cohen P, Hemmings BA: Mechanism of activation of protein kinase B by insulin and IGF-1. EMBO J 1996;15:6541-6551.

82 Shaw M, Cohen P, Alessi DR: The activation of protein kinase B by H2O2 or heat shock is mediated by phosphoinositide 3-kinase and not by mitogen-activated protein kinase-activated protein kinase-2. Biochem J 1998;336:241-246. 